\title{
The Effect of Bevacizumab on Corneal Neovascularization in Rabbits
}

\author{
Wung-Jae Kim, Hee-Ok Jeong, Sung-Kun Chung \\ Department of Ophthalmology, St. Mary's Hospital, The Catholic University of Korea School of Medicine, Seoul, Korea
}

Purpose: To determine the efficacy of topical application and subconjunctival injection of bevacizumab in the treatment of corneal neovascularization.

Methods: Corneal neovascularization was induced with a silk suture of the corneal stroma in 12 rabbits (24 eyes). One week after suturing, four rabbits were treated with topical bevacizumab at $5 \mathrm{mg} / \mathrm{mL}$ (group A) and another four rabbits were treated with topical bevacizumab $10 \mathrm{mg} / \mathrm{mL}$ (group B) in the right eyes twice a day for two weeks. A subconjunctival injection of bevacizumab $1.25 \mathrm{mg} / \mathrm{mL}$ was done in the right eyes of four rabbits (group C). All of the left eyes (12 eyes) were used as controls. The area of corneal neovascularization was measured after one and two weeks, and the concentration of vascular endothelial growth factor (VEGF) in corneal tissue was measured after two weeks.

Results: The neovascularized area was smaller in all treated groups than in the control group $(p<0.001)$. Upon analysis of the neovascularized area, there was no significant difference between groups $A$ and B. However, the mean neovascularized area of group $B$ was significantly smaller than that of group $C$ after two weeks of treatment $(p=0.043)$. The histologic examination revealed fewer new corneal vessels in all treated groups than the control group. The concentration of VEGF was significantly lower in all treated groups compared to the control group $(p<0.01)$, but no difference was shown between treated groups.

Conclusions: Topical and subconjunctival bevacizumab application may be useful in the treatment of corneal neovascularization and further study is necessary.

Key Words: Bevacizumab, Cornea, Neovascularization

Corneal neovascularization is a change of the final result of infectious, inflammatory, traumatic, and metabolic diseases in the cornea, and approximately $4 \%$ of ophthalmic patients have this disease $[1,2]$. Occasionally, corneal neovascularization is helpful to wound healing or the impediment of infection, however it reduces corneal transparency, and thus becomes the cause of visual acuity deterioration and it may become the cause of rejection reaction during corneal transplantation in most cases [2]. To treat and prevent corneal neovascularization, various medical therapies, photodynamic therapy, and laser therapy have been attempted [3-11], but clinically established therapeutic procedures are

Received: January 5, 2009 Accepted: April 16, 2010

Reprint requests to Sung Kun Chung. Department of Ophthalmology, Yeouido St. Mary's Hospital, \#62 Yeouido-dong, Yeongdeungpo-gu, Seoul 150-713, Korea. Tel: 82-2-3779-1114, Fax:82-2-761-6869, E-mail: eyedoc@catholic.ac.kr not available.

Vascular endothelial growth factor (VEGF) has been reported to play a very important role in corneal neovascularization. It has been reported that upon VEGF polymer injection into rat cornea, the formation of neovascularization progressed very rapidly [12], and a higher concentration of VEGF and its receptor was detected than in normal cornea upon examination of human cornea neovascularization [13,14].

Bevacizumab (Avastin; Genentech, San Francisco, CA, USA) is a monoclonal antibody to the VEGF molecule, has been approved by the FDA for the treatment of colorectal cancer, and has been used actively in clinics [15]. In the ophthalmic field, by systemic injection or injection to the vitreous cavity, it has been used effectively for the treatment of age-related macular degeneration as well as diabetic retinal edema or clinically significant macular edema and the results on such treatments have been reported [16-18]. In recent years, bevacizumab has been used to study corneal, conjunctival, and retinal neovascularization with rabbits $[19,20]$. 
In this study, it was examined whether bevacizumab, which has been used actively in retinal applications, is effective on the corneal neovascularization treatment through a simple and safe route, via topical or subconjunctival injection, in rabbit experiments.

\section{Materials and Methods}

\section{Experiment animals}

This study was approved by the Institutional Animal Care and Use Committee of Korea, prior to experiments, and all the in vivo experiment procedures were performed according to regulation of Association for Research in Vision and Ophthalmology for the ophthalmic field and studies on visual function. Twelve New Zealand white rabbits (Samtako, Osan, Korea), weighing between $2.0 \mathrm{~kg}$ and $2.5 \mathrm{~kg}$ were used regardless of their sex; all rabbits were examined prior to surgery and confirmed to have normal cornea.

\section{Induction of corneal neovascularization}

Systemic anesthesia was induced by the intramuscular injection of the mixture of tilemine and zolazepam, Zoletil
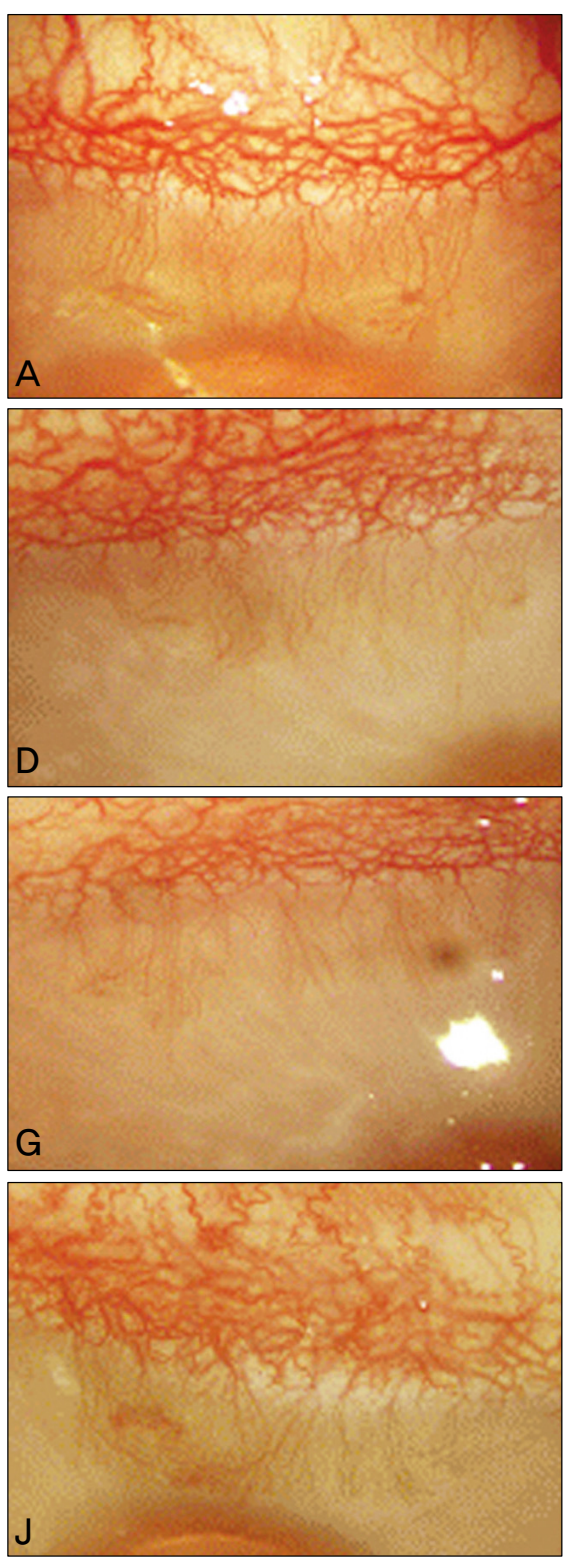
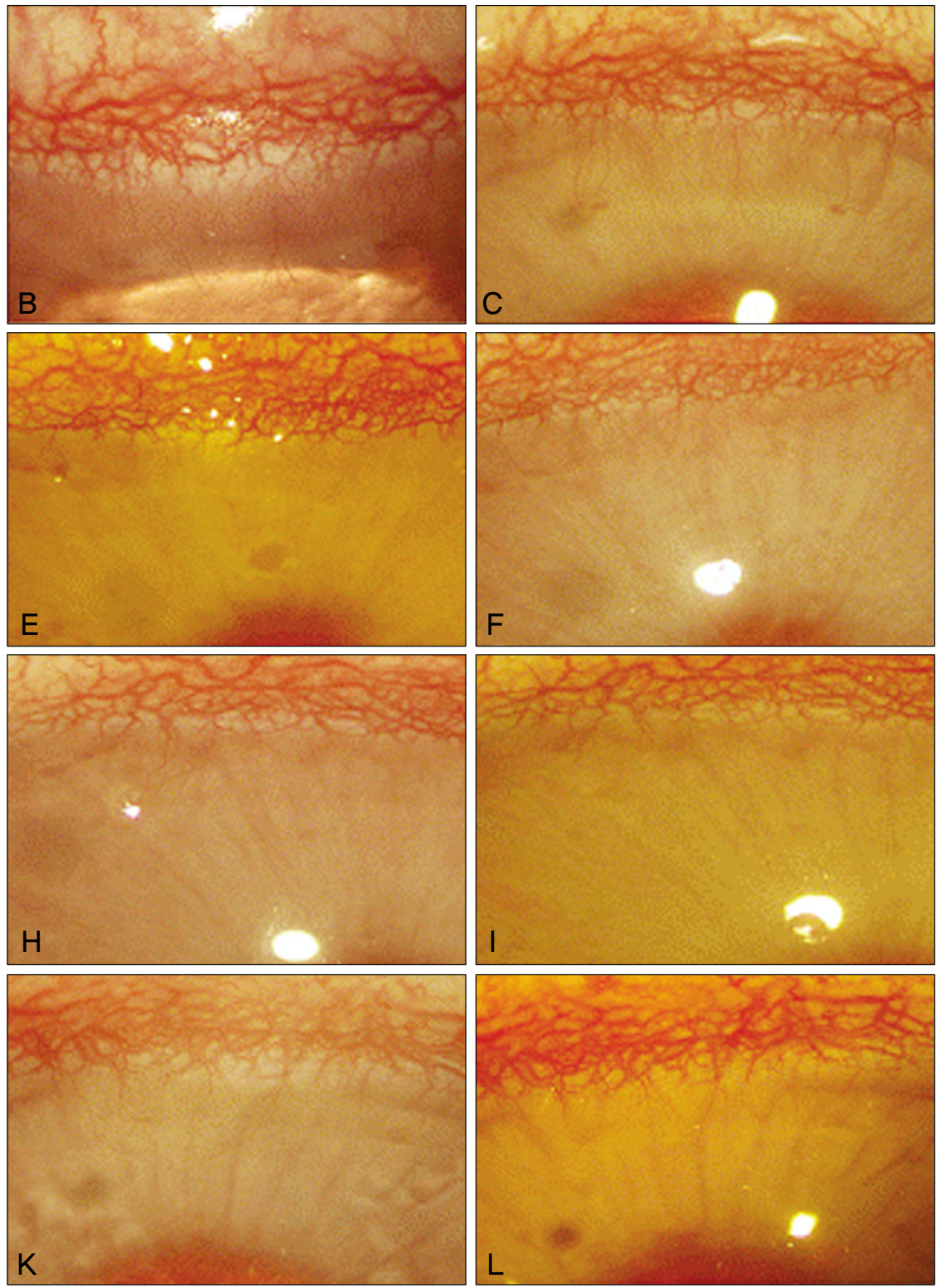

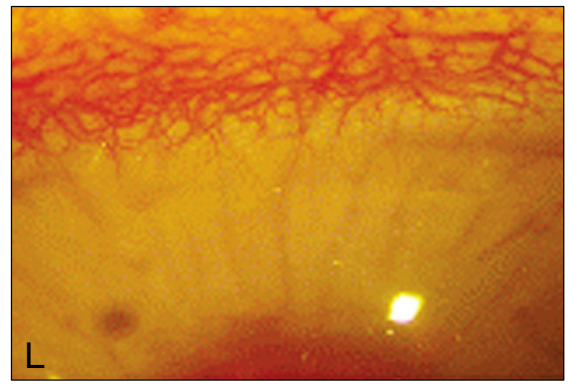

Fig. 1. Microscopic examination of the neovascularized area in the cornea. (A,B,C) Control group. (D,E,F) Group treated with topical 5 $\mathrm{mg} / \mathrm{mL}$ bevacizumab. (G,H,I) Group treated with topical $10 \mathrm{mg} / \mathrm{mL}$ bevacizumab. (J,K,L) Group treated with subconjunctival bevacizumab $1.25 \mathrm{mg}$ injection. (A,D,G,J) Initial neovascularization of the cornea was observed. One week later, neovascularized areas were quite similar in the control group (B), but markedly regressed in the treated groups $(\mathrm{E}, \mathrm{H}, \mathrm{K})$. After two weeks, neovascularized areas were somewhat regressed in the control group $(\mathrm{C})$, but significantly lower than that of treated groups $(\mathrm{F}, \mathrm{I}, \mathrm{L})$. 
(Vibrac, Carros, France), at a $0.2 \mathrm{mg} / \mathrm{kg}$ dose, and topical anesthesia was induced by proparacaine eye drop (Alcaine; Alcon, Fort Worth, TX, USA). In 12 house rabbits (24 eyes), a corneal suture $3 \mathrm{~mm}$ in length passing through the corneal stroma area was performed using 7-0 black silk (Sofsilk; Syneture, Quebec, Canada), at the 12 o'clock direction distanced from the corneal limbus by $1 \mathrm{~mm}$. After suturing, to prevent infection, ofloxacin eye drops (Ocuflox; Samil, Seoul, Korea) were administered four times per day for seven days. One week later, the suture was removed after confirming the sufficient formation of corneal neovascularization.

\section{Treatment of the neovascularization with bevacizumab}

To prevent error caused by the result of systemic absorption, saline was administered to the left eye of all 12 animals without special treatments and used as the control group (12 eyes). Among 12 right eyes, in four eyes cases, a $5 \mathrm{mg} / \mathrm{mL}$ bevacizumab eye drop was administered twice a day for two weeks, and in the other four eyes cases, a $10 \mathrm{mg} / \mathrm{mL}$ bevacizumab eye drop was administered twice a day for two weeks. The remaining four eyes were treated with the subconjunctival injection of $1.25 \mathrm{mg}(0.01 \mathrm{~mL})$ bevacizumab once, and afterward, no other treatments were administered.

\section{The analysis of the neovascularization area}

The picture of the cornea of each experiment group was taken one week and two weeks after treatment with a camera (Contax D-7, Stutgart, Germany) attached to a microscope (S21; Carl Zeiss, Jena, Germany) at 25 times magnification, and the neovascularization area was measured using Axiovision AC software (Carl Zeiss). Considering the area prior to treatment as one, the relative reduction level was calculated and analyzed.

\section{Histological examination and the calculation of VEGF concentration}

Two weeks after treatment, both eyes of 12 animals were extracted and the neovascularization area was cut into halves. The area with neovascularization was prepared as sections, and a histological test was performed. Of corneal sections obtained from each eye, one half was fixed in $10 \%$ neutral formalin, and after a dehydration process, embedded in paraffin. Sections were then prepared, stained with hematoxylin \& eosin, and examined under a biomicroscope (BX-50; Olympus, Tokyo, Japan). From the remaining corneal sections, the area with neovasculatures was measured accurately, and then immediately stored in a $-80^{\circ} \mathrm{C}$ freezer. For these tissues, $1 \mathrm{~mm}$ phenylmethylsulfonylfloride was added to phosphate buffered saline, and then homogenized as $200 \mu \mathrm{L} / \mathrm{g}$ volume. Afterward, the samples were centrifuged at $1,000 \mathrm{~g}$, at $4^{\circ} \mathrm{C}$ for ten minutes, and only the supernatant was used. The concentration of VEGF in tissues was meas- ured by luminometer using the human VEGF immunoassay kit (R\&D System, Minneapolis, MN, USA).

\section{Statistical analysis}

The statistical analysis on the change of vascularization area and VEGF concentration was performed by Mann-Whitney $U$-test and a $p$-value less than 0.05 was considered to be significant.

\section{Results}

\section{Analysis of the area of corneal neovascularization}

In all rabbits, sufficient neovasculature was formed on day seven after corneal suture, while infection, as well as other specific findings, were not detected. Images of the corneal neovascularization area enlarged 25 times were taken using a microscope, and the images prior to surgery, the first week, and the second week were compared and analyzed (Fig. 1).

The area of neovascularization of each group was analyzed and compared, and the result revealed that the groups administered $5 \mathrm{mg} / \mathrm{mL}$ bevacizumab, $10 \mathrm{mg} / \mathrm{mL}$ bevacizumab, and subconjunctival injection of $1.25 \mathrm{mg}$ bevacizumab showed greater reduction of neovascularization area than the control group (Fig. 2).

Comparing the difference between the groups administered $5 \mathrm{mg} / \mathrm{mL}$ bevacizumab eye drop and $10 \mathrm{mg} / \mathrm{mL}$ eye drop, the $p$-value (Mann-Whitney $U$-test) of week 1 was 0.248 , of week 2 was 0.083 ; a significant difference was not

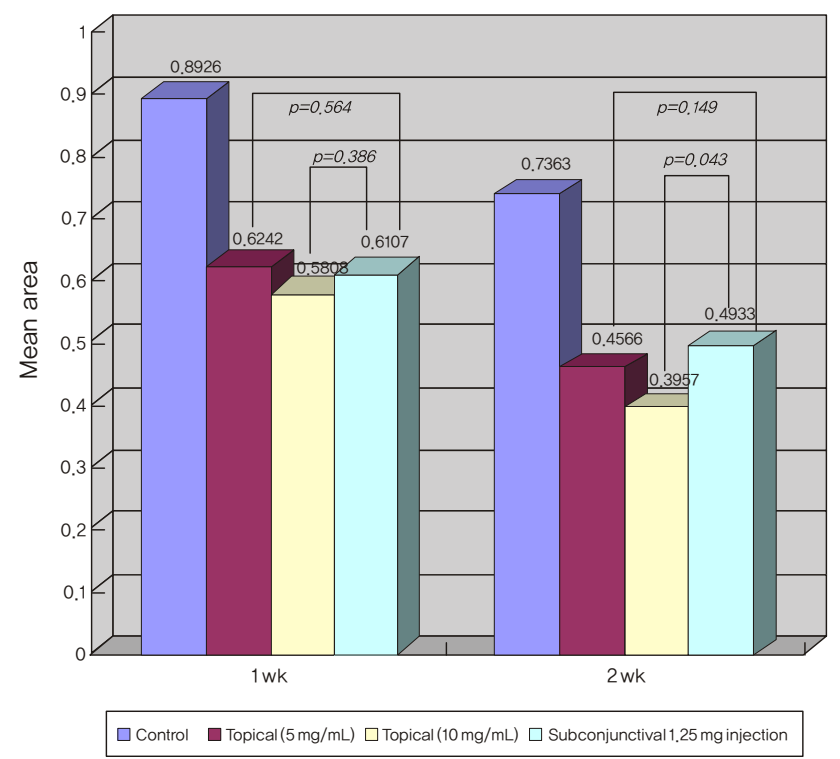

Fig. 2. Mean area of new vessels in the treated and control groups, and $p$-value between topically treated groups and group with subconjunctival injection by Mann-Whitney $U$-test. The group treated with $10 \mathrm{mg} / \mathrm{mL}$ bevacizumab eye drops showed a more significant reduction of neovascularization area than the group treated by subconjunctival injection at the second week. 
shown. Comparing the groups administered bevacizumab eye drop and treated by subconjunctival injection, a significant difference was not detected at the first week. However, the group treated with $10 \mathrm{mg} / \mathrm{mL}$ bevacizumab eye drop showed a more significant reduction of neovascularization area than the group treated by subconjunctival injection at the second week (Fig. 2).

\section{The result of histological examination under light microscope}

The result of corneal section examination two weeks after treatment revealed that a greater neovascularization reduction within the corneal stroma than the control group was shown in the three treated groups. However, among the treated groups, a great difference in the number and area of neovasculatures was difficult to detect. Over the study period, significant injury in the corneal epithelium, stroma, and endothelium, that is, superficial punctate erosion, change of corneal thickness, conjunctival injection or any other type of corneal complication were not observed in all eyes (Fig. 3).

\section{Measure of vascular endothelial growth factor concentration}

The concentration of VEGF in each corneal section was compared, and the result showed that the control group contained $1,284.33 \pm 223.01 \mathrm{pg} / \mathrm{mL}$, while the groups treated with $5 \mathrm{mg} / \mathrm{mL}$ bevacizumab eye drop, $10 \mathrm{mg} / \mathrm{mL}$ eye drop, and subconjunctival injection were shown to be $998.33 \pm 130.93$ $\mathrm{pg} / \mathrm{mL}, 942.00 \pm 32.86 \mathrm{pg} / \mathrm{mL}$, and $968.24 \pm 83.34 \mathrm{pg} / \mathrm{mL}$, respectively. The treated group showed a reduction of significant concentrations in comparison with the control group $(p<0.05)$, but a significant difference of concentrations was not detected among the three treated groups $(p>0.05)$ (Fig. 4).

\section{Discussion}

To maintain the transparency of the cornea, it is very important to maintain avascularity, and for this, the appropriate homeostasis of vascular inhibitor factors and vascular growth factors should be maintained. When the balance of angiogenic factors such as fibroblast growth factor (FGF) and VEGF, and angiogenic suppressors such as angiostatin,
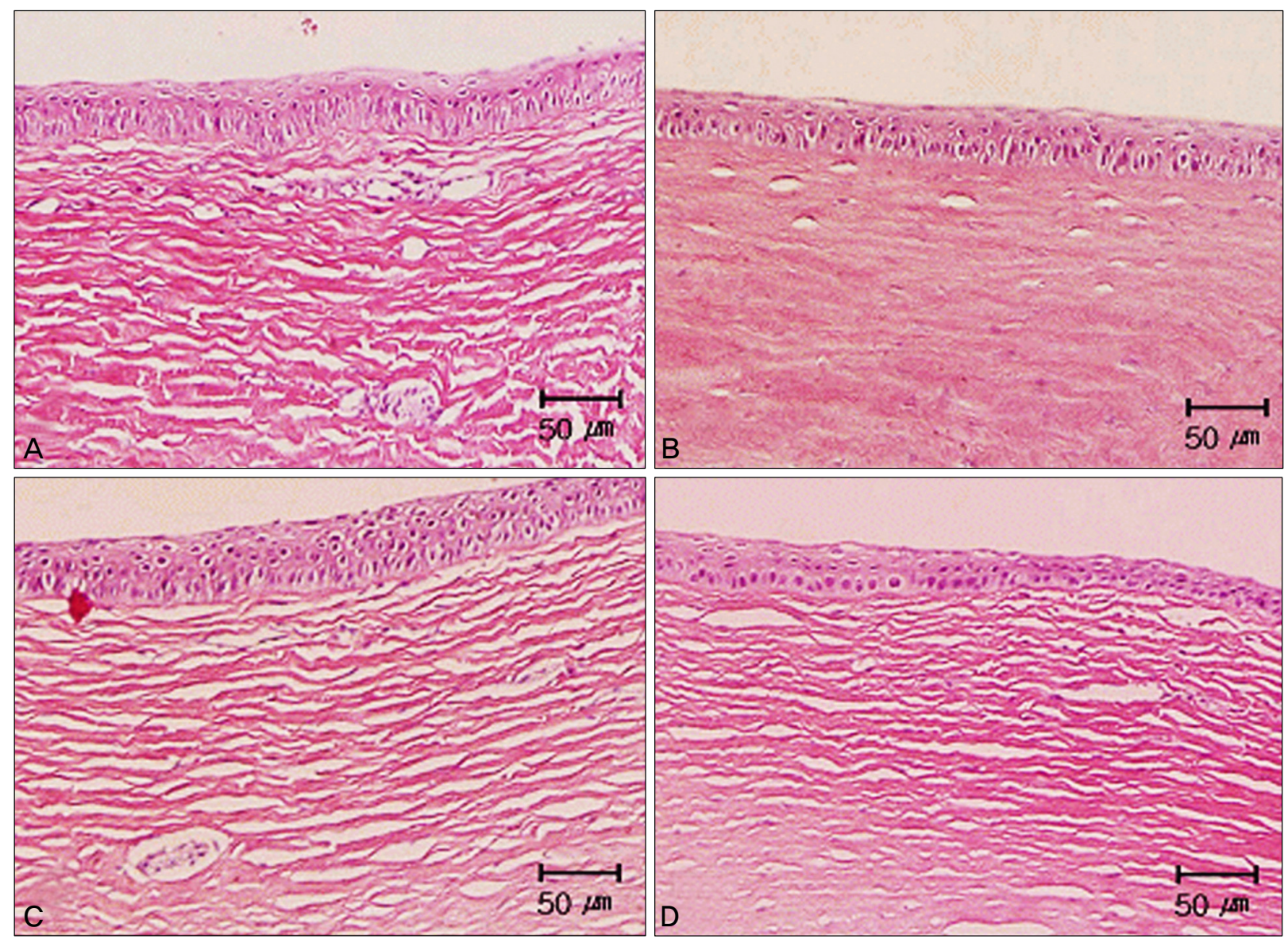

Fig. 3. Light microscopic examination of cornea stained with hematoxylin-eosin. (A) Some new vessels were seen in the stroma of the control group. (B) Some ghost vessels were seen and new vessels were almost regressed in that of groups treated with topical $5 \mathrm{mg} / \mathrm{mL}$ bevacizumab. Markedly regressed new vessels were also observed in that of the group treated with topical $10 \mathrm{mg} / \mathrm{mL}$ bevacizumab (C) and subconjunctival bevacizumab $1.25 \mathrm{mg}$ injection (D). There were no pathologic changes in the stroma, epithelium, and endothelium of all groups. 


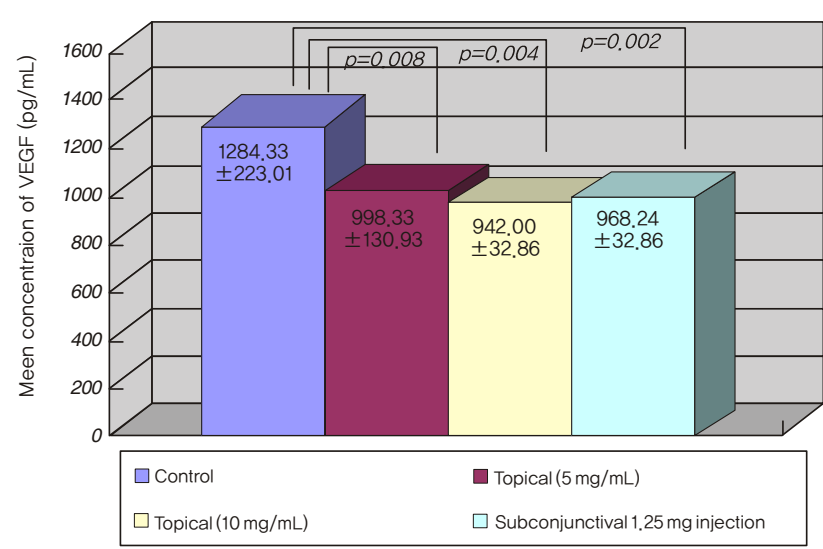

Fig. 4. Mean concentration of vascular endothelial growth factor (VEGF) (pg/mL) in the cornea after two weeks treatment. A $p$-value was estimated by comparing each group with the control group (Mann-Whitney $U$-test). The treated group showed a reduction of significant concentrations in comparison with the control group.

endostatin, and pigment epithelium derived factor (PEDF) are disrupted by diseases, neovascularization develops $[1,21]$.

For the treatment of corneal neovasculature, various drug therapies, laser photocoagulation, and surgical therapy have been attempted, but established therapeutic methods are not currently available. Previously, drug therapies including, steroid [6,8], non-steroid anti-inflammatory agents [3], cyclosporin A [22], thalidomide [3], prolactine [3], methotrexate [7], and angiostatin [4], have been reported. Although steroid eye drop therapy has been used most widely for the treatment and prevention of corneal neovascularization presently, it may cause glaucoma, cataract, infection, and other complications. Hence, its long term use can pose complications [6,8]. Studies attempted to treat corneal neovasculature by the application of angiogenesis suppressor factors such as angiostatin or PEDF, have been conducted, however, they eventually failed. Corneal laser photocoagulation using an argon laser may accompany recurrence of neovascularization, heat injury of adjacent tissues, and the consequent increase of inflammation [9]. As surgical therapy, limbal transplantation [23] or amniotic membrane transplantation [6] has been attempted, however, positive effects were not reported.

Recently, photodynamic treatment using verteporfin also has been used for the treatment of corneal neovascularization. Its short term effect has been reported to be very positive, but its long term effect has not been proven [10,11]. In addition, it has to be injected intravenously and repeated treatments are required, therefore its cost may become extremely high. Because of such diverse problems, verteporfin has been rarely used in clinics to date $[24,25]$.

VEGF has been reported to play a very important role in numerous ophthalmic diseases accompanying neovascularization. VEGF stimulates and accelerates the various processes of neovascularization (protein degradation, proliferation, migration of endothelial cells, and formation of capillary blood vessels). In addition, in recent studies, VEGF-A has shown involvement not only in neovascularization, but also lymphangiogenesis [26,27]. VEGF is not only involved in the regulation of neovascularization, it also has been proven in animal studies that if VEGF were suppressed at the level of mRNA or protein, corneal neovascularization was also decreased [2,14,27].

Bevacizumab is a recombinant monoclonal antibody and it inhibits the binding of VEGF-A to its receptor by binding to VEGF-A [28]. Recently, in the treatment of choroidal neovascularization associated with age-related macular degeneration, positive results of the injection treatment of bevacizumab into the vitreous body have been reported [16-18]. Moreover, the injection of bevacizumab within the vitreous body has been reported to be very effective on the degeneration of the neovasculature in the iris or the iridial corneal margin area [29]. The safety of the drug has been reported to be excellent, and its systemic use has been reported to increase hypertension and thrombosis at a mild level in the treatment of colorectal cancer. However, its probability is very low, and it is thought that in cases of eye drop or subconjunctival injection, the incidence would be substantially decreased $[30,31]$. Also in cases where bevacizumab is injected within the vitreous body, special systemic side effects have not yet been reported [32].

During the injection of bevacizumab within the vitreous body, 1-2 mg is injected, and considering the vitreous body volume $(5.2 \mathrm{~mL})$, the concentration corresponding to $0.2-0.4$ $\mathrm{mg} / \mathrm{mL}$ [16-18]. Considering that the vitreous body is a closed space, it is thought that a concentration higher than this is required for the eye drop to be effective on corneal neovasculature. Therefore, in this study, neovascularization was induced in the cornea of house rabbits, $5 \mathrm{mg} / \mathrm{mL}$ or 10 $\mathrm{mg} / \mathrm{mL}$ bevacizumab eye drop was administered to the eye, and $1.25 \mathrm{mg}$ was used for the injection within the vitreous body.

Our experiments showed that the results of the groups treated with eye drop and subconjunctival injection were significant $(p<0.05)$, but corneal neovasculature could not be completely removed. Considering the several causes of neovasculature formation, administration of bevacizumab twice a day alone via eye drop may not be effective. It is possible that the administered drug is removed by tears, and thus it could not react with all VEGF receptors. In fact, in our experiment, upon analysis of the VEGF concentration of corneal sections, the treatment group showed significantly lower values than the control group. Nevertheless, the VEGF concentration of the control group was still maintained at a constant level (Fig. 4). In other words, even after treatment, a large number of VEGF still remained in tissues. However, even in cases of subconjunctival injection, the result was not significantly different from eye drop treatments, and considering that the half-life of bevacizumab is less than 30 minutes, it appears that removal of the drug via tears may not be a great factor. In addition, the difference of the treatment according 
to eye drop concentrations was shown to be not significant (Fig. 2), therefore additional studies on this variable are also required. Moreover, our findings may be due to the presence of other cytokines (transforming growth factor a and b2; TGF $\mathrm{a}$ and $\mathrm{b} 1$, and FGF involved in corneal neovascularization in addition to VEGF) [21]. In the future, if drugs suppressing these cytokines were developed, a combination therapy may be considered. Third, the treatment period may also be considered. Actually, the eye drop treatment was terminated in two weeks, and the subconjunctival injection was terminated after only one treatment. Nonetheless, at two weeks after treatment, the neovasculature area was definitely smaller than after one week, and at the second week, the outcome of eye drop treatment was significantly better than the subconjunctival treatment $(p=0.043)$. To improve the treatment effectiveness, prolonging the duration of eye drop treatment or repeated subconjunctival treatments may be one option.

Presently, the focus of bevacizumab application to the treatment of corneal neovascularization was the administration route and dose. Regarding the subjects, numerous studies have been performed recently. Furthermore, Bock et al. [33] have proven the fact that in experimental rat models, bevacizumab suppressed not only corneal neovascularization, but also neo-lymphangiogenesis, and both systemic administration and eye drop administration were reported to be effective. In addition, during the experiment processes, toxicity on the cornea was not detected. Manzano et al. [34]. have reported that for the treatment of the neovascularization in the rat cornea, a $4 \mathrm{mg} / \mathrm{mL}$ bevacizumab eye drop was administered, and satisfactory results were obtained. Erdurmus and Totan [35] have reported that in human eyes, $2.5 \mathrm{mg}$ bevacizumab $(0.1 \mathrm{~mL})$ was injected subconjunctivally to two eyes with the neovasculature, which proved effective within one week, and special systemic complications were not detected. Only in one eye case, the degeneration of large blood vessels was not noticeable, which shows the necessity of diverse clinical studies on the dose of bevacizumab, with or without repeated injections, or in combination with other therapies.

In our study, regarding the effectiveness differences according to the eye drop concentration, the effectiveness was shown to not vary by the analysis of the neovasculature area and VEGF concentration, which shows that both routes are effective. However, if a difference between the eye drop treatment and the subconjunctival injection treatment was detected at the second week of treatment, the repeated treatment of the subconjunctival injection should be considered.

In addition to bevacizumab, ranibizumab (Lucentis; Genentech, San Francisco, CA, USA) [36], and pegaptanib sodium (Macugen; Eyetech Pharmaceuticals, New York, NY, USA) [37], are antibodies to VEGF developed recently. While these two drugs were already approved for the ophthalmic application, their shortcoming is excessively high cost. In the future, if the cost problem were solved, the use of these drugs for the treatment of corneal neovascularization should be also considered.
Because bevacizumab suppresses the proliferation of blood vessel and lymphoid tissues, it is hypothesized that it may elevate the survival rate of grafts after corneal transplantation, and it may be used for diverse ophthalmic diseases such as the treatment of herpes keratitis with neovascularization and the prevention of recurrence after the pterygium surgery. Based on our study, it is thought that bevacizumab would be used as a definite supplement therapy for the treatment of corneal neovascularization in the future, and clinical studies on the dose, administration route, administration duration, and frequency are required.

\section{Conflict of Interest}

No potential conflict of interest relevant to this article was reported.

\section{References}

1. Dana MR, Streilein JW. Loss and restoration of immune privilege in eyes with corneal neovascularization. Invest Ophthalmol Vis Sci 1996;37:2485-94.

2. Lee P, Wang CC, Adamis AP. Ocular neovascularization: an epidemiologic review. Surv Ophthalmol 1998;43:245-69.

3. Riazi-Esfahani M, Peyman GA, Aydin E, et al. Prevention of corneal neovascularization: evaluation of various commercially available compounds in an experimental rat model. Cornea 2006;25:801-5.

4. Ambati BK, Joussen AM, Ambati J, et al. Angiostatin inhibits and regresses corneal neovascularization. Arch Ophthalmol 2002;120:1063-8.

5. Shao C, Sima J, Zhang SX, et al. Suppression of corneal neovascularization by PEDF release from human amniotic membranes. Invest Ophthalmol Vis Sci 2004;45:1758-62.

6. Phillips K, Arffa R, Cintron C, et al. Effects of prednisolone and medroxyprogesterone on corneal wound healing, ulceration, and neovascularization. Arch Ophthalmol 1983;101:640-3.

7. Joussen AM, Kruse FE, Volcker HE, Kirchhof B. Topical application of methotrexate for inhibition of corneal angiogenesis. Graefes Arch Clin Exp Ophthalmol 1999;237:920-7.

8. Murata M, Shimizu S, Horiuchi S, Taira M. Inhibitory effect of triamcinolone acetonide on corneal neovascularization. Graefes Arch Clin Exp Ophthalmol 2006;244:205-9.

9. Mendelsohn AD, Stock EL, Lo GG, Schneck GL. Laser photocoagulation of feeder vessels in lipid keratopathy. Ophthalmic Surg 1986; 17:502-8.

10. Primbs GB, Casey R, Wamser K, et al. Photodynamic therapy for corneal neovascularization. Ophthalmic Surg Lasers 1998;29:832-8.

11. Nah HJ, Yoon KC, Im WB, et al. Animal study of photodynamic therapy with verteporfin in corneal neovascularization. J Korean Ophthalmol Soc 2005;46:707-15.

12. Phillips GD, Stone AM, Jones BD, et al. Vascular endothelial growth factor (rhVEGF165) stimulates direct angiogenesis in the rabbit cornea. In Vivo 1994;8:961-5.

13. Cursiefen C, Rummelt C, Kuchle M. Immunohistochemical localization of vascular endothelial growth factor, transforming growth factor alpha, and transforming growth factor betal in human corneas with neovascularization. Cornea 2000;19:526-33.

14. Philipp W, Speicher L, Humpel C. Expression of vascular endothelial growth factor and its receptors in inflamed and vas- 
cularized human corneas. Invest Ophthalmol Vis Sci 2000; 41:2514-22.

15. Presta LG, Chen H, O'Connor SJ, et al. Humanization of an antivascular endothelial growth factor monoclonal antibody for the therapy of solid tumors and other disorders. Cancer Res 1997;57:4593-9.

16. Emerson MV, Lauer AK, Flaxel CJ, et al. Intravitreal bevacizumab (Avastin) treatment of neovascular age-related macular degeneration. Retina 2007;27:439-44.

17. Avery RL, Pieramici DJ, Rabena MD, et al. Intravitreal bevacizumab (Avastin) for neovascular age-related macular degeneration. Ophthalmology 2006;113:363-72.e5.

18. Arevalo JF, Fromow-Guerra J, Quiroz-Mercado H, et al. Primary intravitreal bevacizumab (Avastin) for diabetic macular edema: results from the Pan-American Collaborative Retina Study Group at 6-month follow-up. Ophthalmology 2007; 114:743-50.

19. Woo KJ, Lee K, Choi DG, Choi MY. The effect of subconjunctival injection of bevacizumab after resection of muscle in rabbit models. J Korean Ophthalmol Soc 2010;51:423-9.

20. Kang S, Chung SK. The effect of subconjuctival combined treatment of bevacizumab and triamcinolone acetonide on corneal neovascularization in rabbits. Cornea 2010;29:192-6.

21. Chang JH, Gabison EE, Kato T, Azar DT. Corneal neovascularization. Curr Opin Ophthalmol 2001;12:242-9.

22. Benelli U, Ross JR, Nardi M, Klintworth GK. Corneal neovascularization induced by xenografts or chemical cautery: inhibition by cyclosporin A. Invest Ophthalmol Vis Sci 1997; 38:274-82.

23. Tsai RJ, Li LM, Chen JK. Reconstruction of damaged corneas by transplantation of autologous limbal epithelial cells. $N$ Engl J Med 2000;343:86-93.

24. Yoon KC, Im SK, Oh HJ, Park YG. Two cases of photodynamic therapy with verteporfin in patients with corneal neovascularization. J Korean Ophthalmol Soc 2006;47:13-8.

25. Jun EJ, Rho YJ, Kim YH, Chung SK. The effect of photodynamic therapy with verteporfin retreatment on corneal neovascularization in rabbits. J Korean Ophthalmol Soc 2008; 49:1515-24.
26. Bjorndahl MA, Cao R, Burton JB, et al. Vascular endothelial growth factor-a promotes peritumoral lymphangiogenesis and lymphatic metastasis. Cancer Res 2005;65:9261-8.

27. Hong YK, Lange-Asschenfeldt B, Velasco P, et al. VEGF-A promotes tissue repair-associated lymphatic vessel formation via VEGFR-2 and the alpha1beta1 and alpha2beta1 integrins. FASEB J 2004;18:1111-3.

28. Wang Y, Fei D, Vanderlaan M, Song A. Biological activity of bevacizumab, a humanized anti-VEGF antibody in vitro. Angiogenesis 2004;7:335-45.

29. Yazdani S, Hendi K, Pakravan M. Intravitreal bevacizumab (Avastin) injection for neovascular glaucoma. J Glaucoma 2007;16:437-9.

30. Yoeruek E, Spitzer MS, Tatar O, et al. Safety profile of bevacizumab on cultured human corneal cells. Cornea 2007;26: 977-82.

31. Scappaticci FA, Skillings JR, Holden SN, et al. Arterial thromboembolic events in patients with metastatic carcinoma treated with chemotherapy and bevacizumab. J Natl Cancer Inst 2007;99:1232-9.

32. Quiroz-Mercado H, Ustariz-Gonzalez O, Martinez-Castellanos MA, et al. Our experience after 1765 intravitreal injections of bevacizumab: the importance of being part of a developing story. Semin Ophthalmol 2007;22:109-25.

33. Bock F, Onderka J, Dietrich T, et al. Bevacizumab as a potent inhibitor of inflammatory corneal angiogenesis and lymphangiogenesis. Invest Ophthalmol Vis Sci 2007;48:2545-52.

34. Manzano RP, Peyman GA, Khan P, et al. Inhibition of experimental corneal neovascularisation by bevacizumab (Avastin). Br J Ophthalmol 2007;91:804-7.

35. Erdurmus M, Totan Y. Subconjunctival bevacizumab for corneal neovascularization. Graefes Arch Clin Exp Ophthalmol 2007;245:1577-9.

36. Gaudreault J, Fei D, Rusit J, et al. Preclinical pharmacokinetics of Ranibizumab (rhuFabV2) after a single intravitreal administration. Invest Ophthalmol Vis Sci 2005;46:726-33.

37. Vinores SA. Pegaptanib in the treatment of wet, age-related macular degeneration. Int J Nanomedicine 2006;1:263-8. 